\title{
Apoyo social y resiliencia: predictores de bienestar psicológico en adolescentes con suceso de vida estresante
}

\author{
Social support and resilience: Predictors of psychological \\ well-being on adolescents with stressful life event
}

\author{
Sandybell González Lugo, José Concepción Gaxiola Romero \\ y Edgar René Valenzuela Hernández ${ }^{1}$
}

\begin{abstract}
RESUMEN
Durante la adolescencia existe una mayor vulnerabilidad al desajuste psicológico cuando se experimenta un suceso de vida estresante; sin embargo, existen factores protectores que pueden ayudar al adolescente a gozar de bienestar psicológico a pesar de dichos riesgos. El objetivo del presente estudio fue evaluar el papel del apoyo social y las disposiciones a la resiliencia sobre el bienestar psicológico de adolescentes con sucesos de vida reportados mediante un modelo de trayectorias. Previa firma de un formato de consentimiento informado, 228 estudiantes de bachillerato respondieron una batería de pruebas que evaluaba las variables de interés. En el modelo resultante se muestra que el apoyo social afecta de manera negativa los sucesos de vida experimentados por los adolescentes; a su vez, dichos sucesos se relacionan negativamente con su bienestar psicológico. Otra trayectoria mostró el papel modulador de las disposiciones de resiliencia en el bienestar psicológico de los adolescentes. Se discuten los efectos del apoyo social y las disposiciones a la resiliencia en el bienestar psicológico de la muestra.
\end{abstract}

Palabras clave: Bienestar psicológico; Adolescencia; Resiliencia; Redes de apoyo social.

\begin{abstract}
During adolescence there is increased vulnerability to psychological maladjustment when stressful life events occur. There are, however, protective factors that could help teenagers to achieve or preserve psychological wellbeing despite those risks. The aim of the present study was to evaluate, through a path model, the mediating role of social support and dispositions to resilience on psychological wellbeing of adolescents reporting stressful life events. Previous informed consent, 228 junior college students answered a series of questionnaires. Results show a path model where social support negatively affected stressful life events experienced by adolescents. In turn, stressful life events were negatively associated with their psychological wellbeing. Other path showed the mediating role of dispositions to resilience on adolescents' psychological wellbeing. Results are discussed in terms of the role of social support and dispositions to resilience effects on psychological wellbeing of participants.
\end{abstract}

Key words: Psychological wellbeing; Adolescence; Resilience; Social support networks.

$\mathrm{A}$ partir del enfoque de la psicología positiva, se han desarrollado estudios caracterizados por evaluar conceptos centrados en el bienestar humano, siendo así la contraparte del modelo tradicional de la salud, enfocado únicamente en la patología, y proporcionando así un modelo más positivo e integral de la salud mental (Romero, García-Mas y Brustad, 2009).

El bienestar psicológico puede definirse como la dimensión subjetiva relacionada con la calidad de vida, producida en torno a un juicio valorativo de satisfacción, que tiene relación con el balance entre las expectativas o metas trazadas y los logros obtenidos (Fernández, García y Lorenzo, 2014). Al bienestar

\footnotetext{
${ }^{1}$ Departamento de Psicología y Ciencias de la Comunicación, Universidad de Sonora, Blvd. Luis Encinas y Rosales s/n, Col. Centro, 83000 Hermosillo, Son., México, correos electrónicos: sandybell.gonzalezl@gmail.com, joegaxiola@gmail.com y edgar.valenzuelah@gmail.com. Artículo recibido el 28 de septiembre de 2017 y aceptado el 15 de enero de 2018.
}

Citación: González L., S., Gaxiola R., J.C. y Valenzuela H., E.R. (2018). Apoyo social y resiliencia: predictores de bienestar psicológico en adolescentes con suceso de vida estresante. Psicología y Salud, 28(2), 167-176. 
psicológico se le considera un indicador del funcionamiento del individuo que hace posible el desarrollo de sus capacidades y su crecimiento personal (Vivaldi y Barra, 2012); de igual manera, se le ha relacionado con la percepción de la salud (Fernández et al., 2014; Howell, Kern y Lyubomirsky, 2007; Nitsche, Bitran, Pedrals, Echevarría y Rigotti, 2014). Se ha encontrado que en los adolescentes el bienestar psicológico se correlaciona positivamente con el rendimiento académico y negativamente con la depresión (Robles, Sánchez y Galicia, 2011).

El constructo de bienestar psicológico deriva de la tradición eudaimónica referente al desarrollo del potencial humano (Díaz et al., 2006) y ha sido integrado por Ryff y Singer (2008) en un modelo conformado por las siguientes seis dimensiones:

Autoaceptación: Evaluación que hace el individuo sobre sí mismo y que implica la aceptación de sus fortalezas y debilidades personales.

Relaciones positivas con otros: Desarrollo de fuertes sentimientos de empatía, afecto, capacidad de mantener amistades e identificarse con los demás.

Crecimiento personal: Autorrealización del individuo a partir del crecimiento continuo y la confrontación de nuevos retos en el curso de su vida.

Propósitos de vida: Sentido, dirección e intencionalidad en la vida y existencia de propósitos y significado.

Dominio del entorno: Capacidad de modificar el ambiente a partir de las habilidades físicas y psicológicas.

Autonomía: Sentido de independencia, autodeterminación y autorregulación.

Esta visión del bienestar psicológico se fundamenta en los aportes de la psicología humanista y existencial (Ryff, Keyes y Shmotkin, 2002), cuyo elemento clave es el desarrollo de las potencialidades para alcanzar la autorrealización personal. La visión eudaimónica del bienestar psicológico surge en respuesta a las concepciones hedónicas previas acerca del mismo, enfocadas en la evaluación que las personas hacen del placer, equiparando la felicidad con la satisfacción de los deseos personales (Hauser, Springer y Pudrovska, 2005; Ryff y Singer, 2008); para la concepción eudaimónica, sin embargo, la felicidad consiste en la generación de virtudes personales que conducen al desarrollo y la autorrealización.

Si bien es cierto que en el sentido de autorrealización intervienen algunas características sociodemográficas, como el ingreso y la escolaridad (Hicks, Epperly y Barnes, 2001), el bienestar psicológico no se determina exclusivamente por dichas variables, ya que estas solo refieren la satisfacción o insatisfacción de necesidades importantes en la concepción hedónica. Para la orientación que interesa aquí, esto es, el enfoque eudaimónico, los factores socioeconómicos son simplemente facilitadores del bienestar psicológico dado el efecto mediador que poseen en los aspectos psicológicos y sociales (Hicks et al., 2001; Moyano y Ramos, 2007). Entre los mencionados aspectos que pudieran relacionarse con el bienestar psicológico y que se estudian a partir del enfoque de la psicología positiva, se encuentran la resiliencia y las redes de apoyo social percibidas por el individuo (Morales y González, 2014; Orcasita y Uribe, 2010) (Figura 1).

En dicho enfoque, más centrado en evaluar las fortalezas del individuo que las patologías o comportamientos desajustados (Seligman y Csikszentmihalyi, 2000), el balance de las emociones positivas y negativas es independiente del bienestar del individuo, de manera que una persona puede percibir bienestar psicológico - es decir, tener propósitos de vida, autonomía, relaciones positivas con otros, etc. $-\mathrm{y}$ aun así experimentar emociones negativas o estrés en un momento determinado; de igual manera, pudiera manifestar emociones positivas de manera muy limitada debido a diferentes condiciones generadas por su ambiente (Fernández et al., 2014). Así, en los estudios de bienestar psicológico resulta pertinente evaluar la resiliencia -entendida como la adaptación de los individuos ante situaciones de riesgo (Gaxiola y Frías, 2007) - a fin de determinar su papel mediador en eventos de vida difíciles.

Los adolescentes, al igual que todos los seres humanos, afrontan diversos sucesos de vida considerados negativos, algunos de los cuales pueden resultar muy estresantes y convertirse en factores de riesgo; es esta población la que reporta experimentar una mayor cantidad de sucesos de vida estresantes (Veytia, González-Arratia, Andrade y Oudhof, 2012), los cuales se relacionan 
directamente con la depresión y con otros problemas conductuales y familiares (Heredia, Lucio y Suárez, 2011).

Un suceso de vida se define como una experiencia o acontecimiento que tiene el potencial de producir estrés, por lo que su valoración es fundamental cuando se estudian los procesos adaptativos (Barcelata, Durán y Lucio, 2012). Ante un suceso de vida, es de esperarse que los seres humanos, sobre todo durante la adolescencia, exhiban respuestas de estrés que conllevan una alta probabilidad de desarrollar conductas desadaptadas (Benjet et al., 2009). A su vez, la resiliencia se entiende como un proceso adaptativo mediante el cual es posible hacer frente a las adversidades, siendo uno de sus elementos el conjunto de disposiciones o tendencias que probabilizan la superación de los riesgos; tales disposiciones incluyen tener una actitud positiva de la vida, sentido del humor, perseverancia, religiosidad, autoeficacia, optimismo y orientación a la meta (Gaxiola, Frías, Hurtado, Salcido y Figueroa, 2011).

Además del posible efecto protector de las disposiciones de resiliencia, se ha reportado que el apoyo social en los adolescentes es un factor primordial para que puedan adaptarse a las situaciones de vulnerabilidad (Leiva, Pineda y Encina, 2013). Los estudios acerca del apoyo social se fundamentan en investigaciones experimentales, cuyos resultados muestran que al exponerse sin ese apoyo a los estímulos estresantes, se experimentan efectos adversos que no ocurren cuando se está en compañía de otras personas significativas (Orcasita y Uribe, 2010).

Las redes de apoyo incorporan sistemas funcionalmente delimitados acordes a su significancia, los cuales pueden estar constituidos por ambientes tales como la familia, la escuela, los amigos, los vecinos, los compañeros de trabajo y otros muy diversos miembros de la comunidad, mismos que representan elementos indispensables para la salud, así como también para el ajuste y el bienestar de los individuos (Barquero, Leandro y Sotela 2013; Orcasita y Uribe, 2010) en cuanto que fungen como agentes de protección al proporcionar apoyo emocional e informacional que contribuye a afrontar adecuadamente las situaciones de riesgo en las que se ve inmerso el adolescente (Aguillón, 2011); por consiguiente, la falta de apoyo social o un bajo nivel del mismo repercuten negativamente en el estado de salud del individuo (Aranda y Pando, 2013).

En general, los resultados de las investigaciones en torno al apoyo social lo colocan como un elemento importante de la salud física y psicológica. Aunque la mayoría de estos estudios se han centrado en la población adulta (Ferrada y Zavala, 2014; Vivaldi y Barra, 2012), es importante reconocer que la adolescencia, al ser una etapa de riesgos y vulnerabilidad por los grandes cambios biológicos, psicológicos y sociales que conlleva en un corto periodo de tiempo (Estévez y Emler, 2011), requiere que se evalúen los factores protectores que minimicen durante ella el desarrollo de problemas sociales o de salud. La evaluación de dichas variables permitiría comprender el papel regulador del apoyo social y la resiliencia ante los diversos factores de riesgo y determinar la pertinencia de promover dichos constructos en adolescentes vulnerables para aumentar sus niveles de bienestar psicológico (Figura 1).

Figura 1. Modelo hipotético de trayectorias del bienestar psicológico en estudiantes de bachillerato.

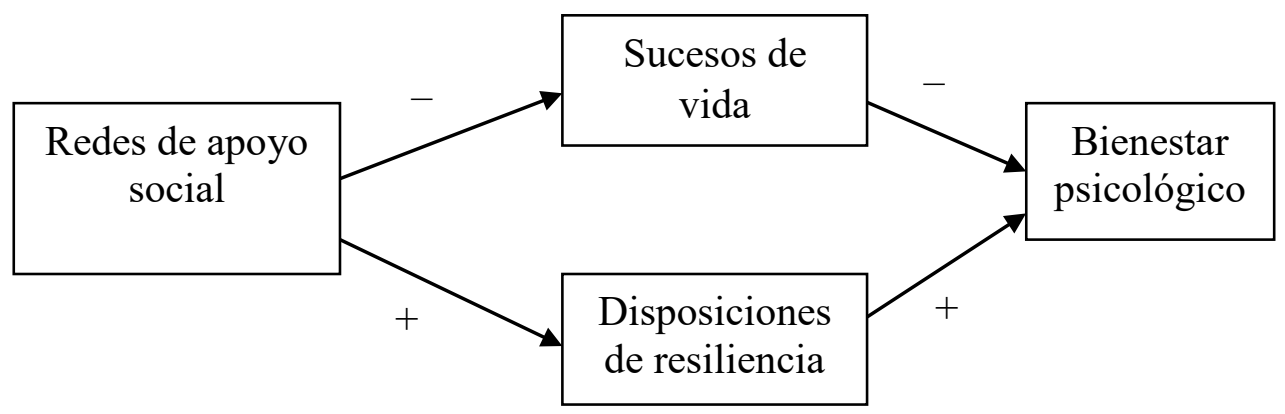


Debido a lo anterior, el objetivo del presente estudio fue evaluar el papel que desempeñan las redes de apoyo social y las disposiciones de resiliencia como predictores del bienestar psicológico de adolescentes que habían experimentado al menos un suceso estresante de vida y que asistían a escuelas preparatorias públicas de una ciudad ubicada al noroeste de México.

\section{MÉTODO}

\section{Participantes}

Se realizó un muestreo no probabilístico e intencional por disponibilidad en una muestra de 709 adolescentes, de los cuales se seleccionó a 228 , quienes reportaron haber presentado al menos un suceso de vida estresante. Lo anterior se justifica debido a que los estudios que miden la resiliencia evalúan el riesgo al cual se debe ajustar el individuo (Gaxiola, 2013). Los adolescentes participantes asistían a dos escuelas públicas de bachillerato de distintos sectores de la localidad, los cuales estaban clasificados con un grado de marginación medio y muy bajo, según los indicadores del Consejo Nacional de Población y Vivienda (2010), lo que permitió incluir jóvenes provenientes de familias de nivel socioeconómico medio-bajo.

De los 228 participantes, 110 fueron hombres y 118 mujeres, todos ellos de nacionalidad mexicana, con una edad promedio de 17.28 años (D.E. = .66).

\section{Instrumentos}

Los sucesos de vida estresantes se evaluaron por medio de una escala adaptada por Torres y Ruiz (2013) para adolescentes. Consta de 34 reactivos que miden sucesos estresantes en las áreas de enfermedad o muerte, separación, sexualidad, actos delictivos y situación económica. Los reactivos muestran cuatro opciones de respuesta en las que se reporta si se ha vivido el suceso y su grado de afectación. Ejemplos de reactivos son los siguientes: "He sido golpeado(a) o duramente castigado(a)", "Murió mi papá (mamá, hermano[a])" y "Fui abusado(a) sexualmente". Para la presente investigación, se seleccionó a quienes reportaron al menos un suceso malo o grave, de manera que se midió la percepción de la gravedad del suceso experimentado. El valor de alfa del instrumento en el estudio piloto fue de .80 .

El apoyo social percibido se evaluó mediante una adaptación de la escala de Redes de Apoyo de Villalobos (2009). La escala original está conformada por 45 reactivos. En una investigación previa, Torres y Ruiz (2013) seleccionaron 19 reactivos para indagar las áreas de apoyo por parte de la familia, amigos, compañeros y otras personas e instituciones. Hay reactivos como "Cuento con mis amigos" y "Tengo personas que me apoyan". La modalidad de respuesta consta de cinco opciones que van de "siempre" a "nunca". El valor del coeficiente alfa en el estudio piloto fue de.78.

El bienestar psicológico se evaluó a través de un inventario con doce ítems, desarrollado en un estudio previo por Gaxiola, Gaxiola, González y Valenzuela (2014), basado en el instrumento elaborado por Ryff (1989). Los reactivos evalúan las áreas de autonomía, relaciones positivas, propósito de vida, autoaceptación, dominio del entorno y crecimiento personal. Algunos ejemplos de reactivos son "Mantiene relaciones cercanas con sus amigos" y "Se siente satisfecho(a) con su vida". Las opciones de respuesta van de 1 ("nada") a 5 ("totalmente"). El valor del coeficiente alfa de la escala reportada por los autores fue de .88.

Las disposiciones a la resiliencia se midieron mediante el Inventario de Resiliencia (IRES) (Gaxiola et al., 2011), conformado por veinte reactivos que evalúan las dimensiones de actitud positiva, sentido del humor, perseverancia, religiosidad, autoeficacia, optimismo y orientación a la meta. Los reactivos se califican a través de cinco opciones de respuesta, que van de 1 ("nada") a 5 ("siempre"). Algunos de los ítems son "Veo lo positivo de la vida y de las cosas que me pasan" y "A pesar de mis problemas procuro ser feliz". Los autores reportaron un valor del coeficiente de alfa de .93

\section{Procedimiento}

Se seleccionaron las dos escuelas referidas cuyas autoridades manifestaron su disposición para participar en el estudio. Una vez obtenidos los permisos, se acudió a las aulas en los horarios de clase. Se 
explicó a los alumnos los objetivos de la investigación $\mathrm{y}$, mediante su consentimiento informado, se les aplicó colectivamente la batería de pruebas. La duración de la evaluación osciló entre 35 y 40 minutos. Para los fines de la presente investigación se seleccionaron únicamente 228 encuestas, correspondientes a los participantes que hubieran reportado al menos un suceso de vida difícil, malo o muy grave.

\section{Análisis de datos}

Los datos se capturaron en el programa estadístico SPSS, versión 21. Se elaboraron los estadísticos descriptivos para las variables categóricas, se obtuvieron los valores del coeficiente alfa de los instrumentos, se sumaron las respuestas medias de las escalas y se conformaron los índices. Se evaluó la relación entre los índices con el coeficiente de correlación de Pearson ( $r$ ). Luego, se exportaron las variables al programa multivariado EQS, versión 6.0, mediante el cual se probaron las trayectorias de los índices conformados como variables. Para determinar la pertinencia del modelo, se utilizó el indicador estadístico chi cuadrado $\left(\chi^{2}\right)$ y los indicadores prácticos Índice Bentler-Bonett de Ajuste Normado (IBAN) y el Índice de Bentler-Bonnett de Ajuste no Normado (IBANN).

\section{RESULTADOS}

En las Tablas 1 y 2 se muestran las características descriptivas de la muestra en términos sociodemográficos y de sucesos de vida experimentados, respectivamente. En la primera de ellas se observa que la mayoría de los adolescentes no trabajaban, vivían con ambos padres y tenían ingresos menores a cinco mil pesos mensuales.

En la Tabla 2 se describe la frecuencia de los sucesos de vida reportados por los adolescentes. Los problemas económicos en la familia, las desilusiones amorosas y el divorcio entre los padres fueron los sucesos más comunes reportados como malos, mientras que los sucesos graves más frecuentes fueron el divorcio y la pérdida del empleo del padre o de la madre.
Tabla 1. Características sociodemográficas de la mues$\operatorname{tra}(\mathrm{N}=228)$.

\begin{tabular}{|c|c|c|}
\hline Variable & $\mathbf{F}$ & $\%$ \\
\hline \multicolumn{3}{|l|}{ Sexo } \\
\hline Masculino & 110 & 48.2 \\
\hline Femenino & 118 & 51.8 \\
\hline \multicolumn{3}{|l|}{ Trabaja } \\
\hline Sí & 49 & 21.5 \\
\hline No & 179 & 78.5 \\
\hline \multicolumn{3}{|l|}{ Vive con } \\
\hline Ambos padres & 124 & 54.4 \\
\hline Madre & 65 & 28.5 \\
\hline Padre & 11 & 4.8 \\
\hline Otro & 25 & 11 \\
\hline \multicolumn{3}{|l|}{ Ingreso familiar* } \\
\hline Menos de $\$ 5,000$ & 85 & 37.3 \\
\hline Entre $\$ 5,000$ y $\$ 10,000$ & 81 & 35.5 \\
\hline Entre $\$ 10,000$ y $\$ 15,000$ & 33 & 14.5 \\
\hline Más de $\$ 15,000$ & 19 & 8.3 \\
\hline \multicolumn{3}{|l|}{ Estado civil de la madre } \\
\hline Casada & 125 & 54.8 \\
\hline Divorciada & 37 & 16.2 \\
\hline Viuda & 12 & 5.3 \\
\hline Soltera & 16 & 7 \\
\hline Unión libre & 28 & 12.3 \\
\hline \multicolumn{3}{|l|}{ Estado civil del padre } \\
\hline Casado & 128 & 56.1 \\
\hline Divorciado & 32 & 14 \\
\hline Viudo & 4 & 1.8 \\
\hline Soltero & 5 & 2.2 \\
\hline Unión libre & 25 & 11 \\
\hline
\end{tabular}

En la Tabla 3 se muestran los resultados de los valores del coeficiente alfa de Cronbach de cada una de las escalas utilizadas en el estudio, las cuales resultaron superiores a .60, por lo que se consideraron aceptables (Nieva y Sorra, 2003).

En la Tabla 4 se presentan las correlaciones entre las variables del estudio, según el coeficiente $r$ de Pearson. Se observa que los sucesos de vida se correlacionaron negativa y significativamente

\footnotetext{
${ }^{2} \mathrm{~A}$ un valor promedio aproximado de 17 pesos por dólar.
} 
Tabla 2. Frecuencia de sucesos de vida reportados como malos y graves.

\begin{tabular}{|c|c|c|c|c|}
\hline \multirow{2}{*}{ SUCESO DE VIDA } & \multicolumn{2}{|c|}{ Malos } & \multicolumn{2}{|c|}{ Graves } \\
\hline & $\mathbf{F}$ & $\%$ & $\mathbf{F}$ & $\%$ \\
\hline Mis padres se separaron o divorciaron. & 50 & 21.8 & 15 & 6.6 \\
\hline Me separé de mis padres para vivir con algún otro familiar, novio(a) o amigo(a). & 8 & 3.5 & 2 & 0.9 \\
\hline Tuve un aborto provocado o mi novia tuvo un aborto provocado. & 1 & 0.4 & 0 & 0.0 \\
\hline Tengo que trabajar además de estudiar. & 12 & 5.2 & 1 & 0.4 \\
\hline Algún familiar o yo padecemos alguna discapacidad. & 14 & 6.1 & 4 & 1.7 \\
\hline Mi familia tiene serios problemas económicos. & 77 & 33.6 & 7 & 3.1 \\
\hline Me embaracé (mujer) o embaracé a mi novia (hombre). & 1 & 0.4 & 0 & 0.0 \\
\hline He sido golpeado(a) o duramente castigado(a). & 12 & 5.2 & 3 & 1.3 \\
\hline Los pleitos entre mis padres empeoran. & 42 & 18.3 & 7 & 3.1 \\
\hline Murió mi padre (madre), hermano(a). & 13 & 5.7 & 16 & 7.0 \\
\hline Mi padre (madre) se fue de la casa. & 34 & 14.8 & 9 & 3.9 \\
\hline Tuve una experiencia sexual con una persona de mi mismo sexo. & 1 & 0.4 & 3 & 1.3 \\
\hline Mi padre (madre) perdió su empleo. & 41 & 17.9 & 15 & 6.6 \\
\hline Padezco alguna enfermedad física o mental grave. & 0 & 0.0 & 1 & 0.4 \\
\hline Algún familiar o yo tenemos problemas relacionados con el alcohol o las drogas. & 32 & 14 & 8 & 3.5 \\
\hline Me expulsaron de la escuela o de algún curso. & 3 & 1.3 & 2 & 0.9 \\
\hline Tuve alguna experiencia sexual desagradable o traumática. & 5 & 2.2 & 4 & 1.7 \\
\hline Fui golpeado(a) por algún miembro de mi familia. & 9 & 3.9 & 4 & 1.7 \\
\hline Tuve un accidente que me dejó cicatrices. & 10 & 4.4 & 7 & 3.1 \\
\hline Uno de mis padres o hermanos(as) está en la cárcel. & 6 & 2.6 & 1 & 0.4 \\
\hline Intenté quitarme la vida. & 6 & 2.6 & 6 & 2.6 \\
\hline Subí o bajé mucho de peso. & 39 & 17 & 6 & 2.6 \\
\hline Tengo problemas de fe (crisis religiosa). & 18 & 7.9 & 6 & 2.6 \\
\hline Tuve un fracaso (en la escuela). & 45 & 19.7 & 7 & 3.1 \\
\hline Fui abusada $(0)$ sexualmente. & 1 & 0.4 & 2 & 0.9 \\
\hline Reprobé un examen o un curso importante. & 59 & 25.8 & 16 & 0.7 \\
\hline Tengo algún familiar desaparecido (no se sabe dónde está). & 10 & 4.4 & 4 & 1.7 \\
\hline Me suspendieron de la escuela. & 9 & 3.9 & 1 & 0.4 \\
\hline Reprobé o repetí un año escolar. & 14 & 6.1 & 6 & 2.6 \\
\hline Mi padre, madre o hermanos padecen alguna enfermedad fisica, mental grave o importante. & 17 & 7.4 & 1 & 0.4 \\
\hline Tuve alguna desilusión amorosa. & 59 & 25.8 & 13 & 5.7 \\
\hline Algún familiar o yo mismo vivimos un asalto violento o un secuestro. & 20 & 8.7 & 5 & 2.2 \\
\hline Me enteré de que me adoptaron. & 1 & 0.4 & 0 & 0.0 \\
\hline
\end{tabular}

con el bienestar psicológico y las redes de apoyo social; estas se asociaron de forma positiva y significativa con el bienestar psicológico y las disposiciones de resiliencia, y a su vez las disposiciones de resiliencia se correlacionaron con el bienestar psicológico.
Tabla 3. Valores del coeficiente alfa de Cronbach en las escalas $(\mathrm{N}=228)$.

\begin{tabular}{|l|c|}
\hline \multicolumn{1}{|c|}{ Escalas } & $\boldsymbol{\alpha}$ \\
\hline Sucesos de Vida & .80 \\
\hline Redes de Apoyo & .75 \\
\hline Bienestar Psicológico & .86 \\
\hline Disposiciones de Resiliencia & .90 \\
\hline
\end{tabular}


Tabla 4. Correlaciones de $\mathrm{r}$ de Pearson entre las variables del estudio.

\begin{tabular}{|l|c|c|c|c|}
\hline \multicolumn{1}{|c|}{ Variables } & Sucesos de vida & $\begin{array}{c}\text { Redes } \\
\text { de apoyo }\end{array}$ & $\begin{array}{c}\text { Bienestar } \\
\text { psicológico }\end{array}$ & $\begin{array}{c}\text { Disposiciones } \\
\text { de resiliencia }\end{array}$ \\
\hline Sucesos de vida & 1 & & & \\
\hline Redes de apoyo & $-.253^{* *}$ & 1 & & \\
\hline Bienestar psicológico & $-.193^{* *}$ & $.532^{* *}$ & 1 & \\
\hline Disposiciones de resiliencia & -.087 & $.457^{* *}$ & $.619^{* *}$ & 1 \\
\hline
\end{tabular}

* La correlación es significativa al nivel 0.05 (bilateral).

** La correlación es significativa al nivel 0.01 (bilateral).

La Figura 2 muestra las trayectorias obtenidas en el análisis, donde las redes de apoyo social afectan de manera negativa a los sucesos de vida, y estos a su vez se relacionan de manera negativa con el bienestar psicológico; las redes de apoyo social lo hicieron de manera positiva con las disposiciones de resiliencia, y estas a su vez con el bienestar psicológico.

Figura 2. Modelo de trayectorias del bienestar psicológico en estudiantes de bachillerato.

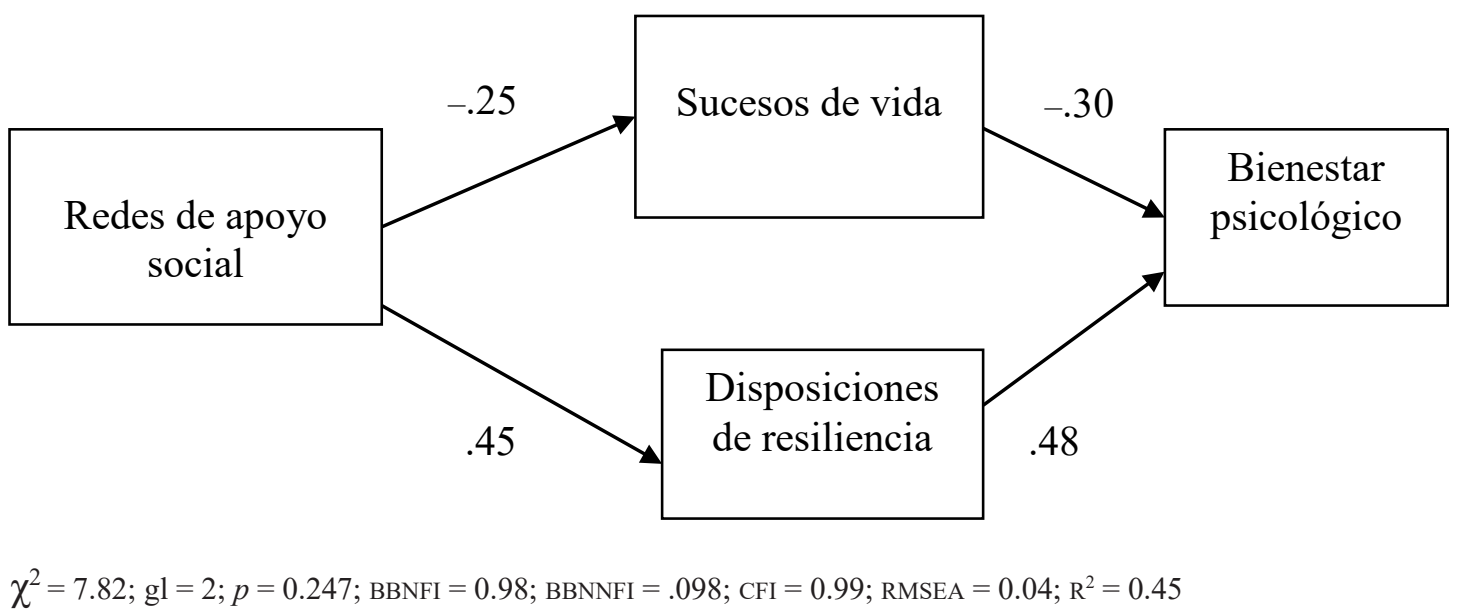

Los indicadores de bondad de ajuste del modelo de bienestar psicológico (BBNFI, BBNNFI y CFI) fueron cercanos a 1.0, y el indicador $\chi^{2}$ obtuvo un valor alto y no significativo (7.82), con un residuo de cuadrados mínimos (RMSEA) menor a 0.05 . Ello implica que el modelo teórico conformado por las relaciones según la literatura se ajusta a los datos, de manera que el modelo sintético no difiere significativamente del modelo saturado, formado por las relaciones entre las variables (Bentler, 2006). El bienestar psicológico de los adolescentes resultó tener una varianza explicada de $45 \%$ $\left(\mathrm{R}^{2}=0.45\right)$.

\section{DISCUSIÓN}

En el modelo resultante se observan las relaciones entre las variables estudiadas y su impacto sobre el bienestar psicológico de los adolescentes. Las redes de apoyo social afectaron de manera negativa la gravedad percibida de los sucesos de vida estresantes, hallazgo que difiere de lo reportado por Barra, Cerna, Kramm y Véliz (2006), en que el apoyo social no fue suficiente para moderar el estrés generado por un suceso de vida; sin embargo, concuerda con otros hallazgos (Lewis, Byrd y Ollendick, 2012; Peng et al., 2012), donde el apoyo social se relacionó de manera inversa con los sucesos de vida negativos. Tal discrepancia en las relaciones puede deberse a que el apoyo social por sí solo no es suficiente para proteger a los adolescentes de los sucesos de vida estresantes (Hammack, Richards, Luo, Edlynn y Roy, 2004). La relación entre ambas variables se puede explicar a través de la percepción de apoyo instrumental y emocional por parte de otras personas, la que puede reducir el estrés que generan los sucesos de 
vida en virtud de que se dispone de más herramientas para afrontar mejor dichos eventos.

Por otro lado, las redes de apoyo social afectaron positivamente las disposiciones a la resiliencia de los participantes; es decir, contar con personas significativas en diferentes contextos se relacionó con la propensión a superar los riesgos (Leiva et al., 2013), lo que implica que tener una visión positiva de la vida, plantearse metas o tener sentido del humor, entre otras tendencias, posibilitan la adaptación del individuo (Gaxiola et al., 2011). Esta relación entre el apoyo social y la resiliencia, medida esta como la superación de las adversidades, es consistente con otras investigaciones hechas con distintas poblaciones: niños (Valenzuela, 2014), adolescentes (Villalobos, Arévalo y Rojas, 2012), adultos (Crespo y Fernández, 2015) y adultos mayores. Por consiguiente, el apoyo social es un recurso importante durante todo el transcurso de la vida.

Por su parte, las disposiciones de resiliencia que derivan de las redes de apoyo social afectaron positivamente el bienestar psicológico de los adolescentes. Este hallazgo demuestra que la resiliencia tiene efectos positivos sobre el comportamiento al mitigar los efectos de los factores de riesgo, tal y como se ha definido este constructo (Cabanyes, 2010). Así, una persona con sentido del humor, perseverancia y actitud positiva de la vida, entre otras tendencias, muestra mayores niveles de bienestar psicológico dado que es más probable que valore positivamente sus relaciones con otros, perciba un dominio del ambiente y experi- mente crecimiento personal, autonomía y demás componentes del modelo de bienestar psicológico (Ryff y Singer, 2008).

El apoyo social se relacionó indirectamente con el bienestar psicológico mediado por las disposiciones de resiliencia, de manera que la existencia de otras personas significativas que brindan apoyo instrumental y afectivo es un recurso fundamental para la salud psicológica. La relación entre ambas variables ha sido reportada por Orcasita y Uribe (2010), en una revisión teórica sobre el valor del apoyo social en el bienestar psicológico de adolescentes en riesgo.

Otro hallazgo importante es que a pesar de que la mayoría de los adolescentes del estudio provinieron de familias con ingresos económicos bajos, no los limitó al reportar bienestar psicológico, lo que apoya los modelos de bienestar basados en la autorrealización personal, más que en la satisfacción o insatisfacción de las necesidades (Ryff y Singer, 2008).

A partir del modelo resultante, se recomienda promover las redes de apoyo social en los adolescentes con características similares a las de la muestra utilizada para disminuir la gravedad percibida de los sucesos de vida estresante, y a la vez fomentar las disposiciones de resiliencia y el bienestar psicológico. Para futuros estudios, se recomienda un análisis más detallado de los sucesos de vida experimentados, a fin de diferenciar el tipo de vivencias que tiene mayor o menor peso en el bienestar psicológico, y los tipos de sucesos específicos que influyen en las redes de apoyo social.

\section{REFERENCIAS}

Aguillón, E. (2011). Caracterización de bullying y redes de apoyo social en adolescentes de una institución educativa pública de Floridablanca-Santander. Tesis inédita de licenciatura. Bucaramanga (Colombia): Universidad Pontificia Bolivariana.

Aranda, C. y Pando, M. (2013). Conceptualización del apoyo social y las redes de apoyo social. Revista de Investigación en Psicología, 16(1), 223-245. Recuperado de http://revistasinvestigacion.unmsm.edu.pe/index.php/psico/article/viewFile/3929/3153.

Barcelata B., E., Durán, C. y Lucio G.M., M.E. (2012). Valoración subjetiva de los sucesos de vida estresantes en dos grupos de adolescentes de zonas marginadas. Salud Mental, 35(6), 513-520. Recuperado de http://www.scielo.org.mx/scielo.php?script=sci_arttextypid= S0185-33252012000600009ylng = esytlng $=$ es.

Barquero, P., Leandro, M. y Sotela, G. (2013). Determinantes socioambientales de la salud mental. En Grupo de Investigación en Salud Mental y Bienestar (Ed.): Estado del arte de la investigación en salud mental en Costa Rica (pp. 26-40). San José: Editor.

Barra A., E., Cerna C., R., Kramm M., D. y Véliz V., V. (2006). Problemas de salud, estrés, afrontamiento, depresión y apoyo social en adolescentes. Terapia Psicológica, 24(1), 55-61.

Benjet, C., Borges, G., Medina-Mora, M.E., Zambrano, J., Cruz, C. y Méndez, E. (2009). Descriptive epidemiology of chronic childhood adversity in Mexican adolescents. Journal of Adolescent Health, 45(5), 483-489. doi: 10.1016/j.jadohealth.2009.03.002. 
Apoyo social y resiliencia: predictores de bienestar psicológico en adolescentes con suceso de vida estresante

Bentler, P.M. (2006). EQS 6 Structural Equations Program Manual. Encino, CA: Mulivariate Software.

Cabanyes, J. (2010). Resiliencia: una aproximación al concepto. Revista de Psiquiatría y Salud Mental, 3(4), 145-151. doi: 10.1016/j. rpsm.2010.09.003.

Consejo Nacional de Población y Vivienda (2010). Índice de marginación por localidad. México: CONAPO. Recuperado de http://conapo.gob.mx/en/CONAPO/Indice_de_Marginacion_por_Localidad 2010.

Crespo, M. y Fernández L., V. (2015). Resiliencia en cuidadores familiares de personas mayores dependientes. Anales de Psicología, 31(1), 19-27. doi: 10.6018/analesps.31.1.158241.

Díaz, D., Rodríguez C., R., Blanco, A., Moreno J., B., Gallardo, I., Valle, C. y van Dierendock, D. (2006). Adaptación española de las escalas de bienestar psicológico de Ryff. Psicothema, 18(3), 572-577.

Estévez, E. y Emler, N.P. (2011). Assessing the links among adolescent and youth offenders, antisocial behavior, victimization, drug use, and gender. International Journal of Clinical and Health Psychology, 11(2), 269-289.

Fernández, A., García, C. y Lorenzo, A. (2014). Consideraciones acerca del bienestar psicológico. Revista Electrónica de Psicología Iztacala, 17(3), 1108-1146.

Ferrada, L. y Zavala, M. (2014). Bienestar psicológico: adultos mayores activos a través del voluntariado. Ciencia y Enfermería, 20(1). 123-130. doi: 10.4067/S0717-95532014000100011.

Gaxiola, J.C. (2013). Aportaciones conceptuales al estudio de la resiliencia. En J. C. Gaxiola y J. Palomar (Coords): Estudios de resiliencia en América Latina (v. 2, pp. 1-17). México: Pearson.

Gaxiola, J.C. y Frías, M. (2007). Los factores protectores y la adaptabilidad al abuso infantil: un estudio con madres mexicanas. Revista Mexicana de Psicología (número especial: Memoria del XV Congreso Mexicano de Psicología), 222-223.

Gaxiola, J.C., Frías A., M., Hurtado A., M.F., Salcido N., L.C. y Figueroa F., M. (2011). Validación del Inventario de Resiliencia (IRES) en una población del noroeste de México. Enseñanza e Investigación en Psicología, 16(1), 73-83.

Gaxiola, J.C., Gaxiola, E., González, S. y Valenzuela E., R. (2014). Trayectoria adaptativa de la crianza positiva materna [CD-ROM]. Memorias del Congreso Mexicano de Psicología (pp. 557-559), Puerto Vallarta (México), 11-13 de octubre.

Hammack, P.L., Richards, M.H., Luo, Z., Edlynn, E.S. y Roy, K. (2004). Social support factors as moderators of community exposure among inner city African American young adolescents. Journal of Clinical Child Psychology and Psychiatry, 33(3), 450-462. doi: 10.1207/s15374424jccp3303_3.

Hauser, R., Springer, K. y Pudrovska, T. (2005) Temporal structures of psychological well-being: continuity or change? Proceedings of the Meetings of the Gerontological Society of America. Orlando, FLO: GSA.

Heredia A., M.L., Lucio G.M., M.E. y Suárez C., L.E. (2011). Depresión y sucesos de vida estresantes en adolescentes. Revista Latinoamericana de Medicina Conductual, 1(2), 49-57.

Hicks, J., Epperly, L. y Barnes, K. (2001). Gender, emotional support, and well-being among the rural elderly. Sex Roles, 45, 15-30. doi: 10.1023/A:1013056116857.

Howell, R., Kern, M. y Lyubomirsky, S. (2007). Health benefits: Meta-analytically determining the impact of well-being on objective health outcomes. Health Psychology Reviews, 1(1), 83-136. doi: 10.1080/17437190701492486.

Leiva, L., Pineda, M. y Encina, Y. (2013). Autoestima y apoyo social como predictores de la resiliencia en un grupo de adolescentes en vulnerabilidad social. Revista de Psicología, 22(2), 111-123. doi: 10.5354/0719-0581.2013.30859.

Lewis, K., Byrd, D. y Ollendick, T. (2012). Anxiety symptoms in African-American and Caucasian youth: Relations to negative life events, social support, and coping. Journal of Anxiety Disorders, 26, 32-39. doi: 10.1016/j.janxdis.2011.08.007.

Morales, N. y González, A. (2014). Resiliencia, autoestima, bienestar psicológico y capacidad intelectual en estudiantes de cuarto medio de bien rendimiento en liceos vulnerables. Estudios Pedagógicos, 40(1), 215-228. doi: 10.4067/S0718-07052014000100013.

Moyano, E. y Ramos, N. (2007). Bienestar subjetivo: midiendo la satisfacción vital, felicidad y su relación con variables sociodemográficas en la población chilena de la región maule. Universum, 22(2), 177-193. doi: 10.4067/S0718-23762007000200012.

Nieva, V.F. y Sorra, J. (2003). Safety culture assessment: a tool for improving patient safety in healthcare organizations. Quality Safe Health Care, 12(Supl), 17-23. doi: 10.1136/qhc.12.suppl_2.ii17.

Nitsche, M.P., Bitran, M., Pedrals, N., Echeverria, G. y Rigotti, A. (2014). Recursos psicosociales positivos y salud cardiovascular. Revista Médica de Chile, 142(10), 1316-1323. doi: 10.4067/S0034-98872014001000012.

Orcasita, L. y Uribe, A. (2010). La importancia del apoyo social en el bienestar de los adolescentes. Psicología: Avances de la Disciplina, 4(2), 69-82.

Peng, L., Zhang, J., Li, M., Li, P., Zhang, Y., Zuo, X., Miao, Y. y Xu, Y. (2012). Negative life events and mental health of Chinese medical students: The effect of the resilience, personality, and social support. Psychiatry Research, 196, 138-141. doi: 10.1016/j. psychres.2011.12.006.

Robles, F.J., Sánchez, A. y Galicia, I.X. (2011). Relación del bienestar psicológico, depresión y rendimiento académico en estudiantes de secundaria. Psicología Iberoamericana, 19(2), 27-37.

Romero, A., García-Mas, A. y Brustad, R. (2009). Estado del arte y perspectiva actual del concepto de bienestar psicológico en psicología del deporte. Revista Latinoamericana de Psicología, 41(2), 335- 347. 
Ryff, C. (1989). Happiness is everything, or is it? Explorations on the meaning of psychological well-being. Journal of Personality and Social Psychology, 57, 1069-1081. doi: 10.1037/0022-3514.57.6.1069.

Ryff, C., Keyes, C. y Shmotkin, D. (2002). Optimizing well-being: the empirical encounter of two traditions. Journal of Personality and Social Psychology, 82(6), 1007-1022. doi: 10.1037//0022-3514.82.6.1007.

Ryff, C. y Singer, H. (2008). Know thyself and become what you are: A eudaimonic approach to psychological well-being. Journal of Happiness Studies, 9, 13-39. doi: 10.1007/s10902-006-9019-0.

Seligman, M.E.P. y Csikszentmihalyi, M. (2000). Positive psychology: an introduction. American Psychologist, 55(1), 5-14. doi: 10.1037/0003-066X.55.1.5.

Torres, M. y Ruiz, A. (2013). Habilidades de resiliencia e estudiantes de educación media superior del Estado de México. En J. C. Gaxiola y J. Palomar (Coords.): Estudios de resiliencia en América Latina (v. 2, pp. 53-69). México: Pearson.

Valenzuela, E. (2014). Influencia del apoyo social en niños testigos de violencia doméstica. Tesis inédita de licenciatura. Hermosillo (México): Universidad de Sonora.

Veytia, M., González-Arratia, N.I., Andrade P., P. y Oudhof, H. (2012). Depresión en adolescentes: el papel de los sucesos vitales estresantes. Salud Mental, 35(1), 37-43.

Villalobos, A. (2009). Desarrollo de una medida de resiliencia para establecer el perfil psicosocial de los adolescentes resilientes que permita poner a prueba el modelo explicativo de resiliencia basado en variables psicosociales. Tesis inédita de especialidad. San José de Costa Rica: Universidad de Costa Rica.

Villalobos G., H., Arévalo, C. y Rojas, F. (2012). Adaptación del Inventario de Resiliencia ante el Suicidio (SRI-25) en adolescentes y jóvenes de Colombia. Revista Panamericana de Salud Pública, 31(3), 233-239. doi: 10.1590/S1020-49892012000300008.

Vivaldi, F. y Barra, E. (2012). Bienestar psicológico, apoyo social percibido y percepción de salud en adultos mayores. Terapia Psicológica, 30(2), 23-29. doi: 10.4067/S0718-48082012000200002. 